\title{
Avaliação de diferentes dietas merídicas sobre a emergência de adultos de Ctenocephalides felis felis (Siphonaptera : Pulicidae)
}

\author{
Evaluation of different meridic diets upon adult emergence of Ctenocephalides felis felis \\ (Siphonaptera: Pulicidae)
}

\author{
Vanessa Paulino da Cruz Vieira ${ }^{1 *}$; Pedro Ivan Fazio Junior ${ }^{2}$; Guilherme Gomes Verocai ${ }^{3}$; \\ Thais Ribeiro Correia ${ }^{4}$; Fabio Barbour Scott ${ }^{5}$
}

${ }^{1}$ Departamento de Parasitologia Animal, Instituto de Veterinária, Universidade Federal Rural do Rio de Janeiro - UFRRJ

${ }^{2}$ Médico Veterinário Autônomo

${ }^{3}$ Department of Ecosystem and Public Health, Faculty of Veterinary Medicine, University of Calgary - UCalgary

${ }^{4}$ Departamento de Parasitologia Animal IV, Universidade Federal Rural do Rio de Janeiro - UFRRJ

${ }^{5}$ Departamento de Parasitologia Animal IV, Universidade Federal Rural do Rio de Janeiro - UFRRJ

Recebido em 6 de Julho de 2009

Aceito em 14 de Agosto de 2009

\section{Resumo}

O objetivo do presente trabalho foi avaliar a influência de dietas merídicas compostas por sangue de diferentes espécies animais sobre a emergência de adultos da pulga Ctenocephalides felis felis. Foram utilizadas seis dietas artificiais contendo areia e sangue desidratado de cão, boi, coelho e galinha, acrescidas ou não com farelo de trigo. Foram realizadas seis repetiçốes contendo dez ovos de C. f. felis para cada dieta. Após 25 dias de incubação, os ovos foram quantificados e avaliados quanto à emergência de adultos. O número de pulgas emergidas para dietas com sangue de boi, cấo, coelho e galinha, com areia e farelo de trigo, foi de aproximadamente oito pulgas. Para as dietas contendo apenas sangue de boi ou de cáo e areia, essa emergência foi de aproximadamente uma pulga para ambas. Conclui-se que a origem do sangue empregado na elaboração da dieta não interfere significativamente no percentual de emergência de adultos de C.f.felis. Portanto, pode-se optar pela espécie animal disponível para o preparo da dieta artificial, aliada à suplementaçáo com farelo de trigo, para suprir uma eventual perda nutricional decorrente da desidrataçáo do sangue.

Palavras-chave: pulga, ovos, ciclo biológico.

\begin{abstract}
The objective of the present study was to evaluate the influence of meridic diets composed by blood from different animal species upon the adult emergence of Ctenocephalides felis felis fleas. Six artificial diets containing dried blood of cattle (standard), dogs, rabbit and chicken, combined with sand or sand/wheat bran were prepared. For each diet six samples containing ten $C$. f. felis eggs were evaluated. After 25 days of incubation, samples were assessed for adult emergence. The number of emerge fleas for diets composed by bovine, canine, rabbit and chicken combined with sand/ wheat bran was aproximally eight fleas. Diets composed by bovine and canine combined with sand, this emergence was aproximally one flea, for both. It can be concluded that the blood origin do not alter significantly $C$. f. felis adult emergence rate. Therefore, blood from any available animal species can be used for artificial diet formulation, combined with wheat bran in order to supply any nutritional losses occurred during blood dehydration.
\end{abstract}

Keywords: flea, eggs, biological cycle.

\footnotetext{
*Autor para correspondência: Vanessa Paulino da Cruz Vieira

Discente de Doutorado, Curso de Pós-Graduação em Ciências Veterinárias

Departamento de Parasitologia Animal, Instituto de Veterinária

Universidade Federal Rural do Rio de Janeiro - UFRRJ,

CEP 23890-000, Rio de Janeiro - RJ, Brasil

e-mail: cruzvp@ufrrj.br
} 
A pulga Ctenocephalides felis felis (BOUCHÉ, 1835) é um ectoparasito de grande importância em medicina veterinária e saúde pública, sendo responsável pela transmissão de diversos patógenos, como cestóides, protozoários, vírus e rickéttsias, aos animais e ao homem (VOBIS et al., 2003; RUST, 2005; PEREZ-OSORIO et al., 2008).

No que diz respeito à alimentação artificial desse ectoparasito, sabe-se que as larvas devem se alimentar de sangue e levedura seca, ou algo similar que contêm nutrientes (HSU et al., 2002). Para complementar seu regime metódico de alimentação, ou seja, sua dieta, as larvas podem praticar o canibalismo, alimentando-se de ovos de sua própria espécie (LAWRENCE; FOIL, 2002).

Em 1997, Linardi et al. 1997, demonstraram que sangue desidratado de camundongo e pombo também pode ser utilizado na dieta larvária, mas que são escassos os estudos nesse âmbito. A utilização de sangue desidratado de outras espécies de mamíferos e até mesmo de aves, em dietas artificiais, faz-se necessária para o desenvolvimento de dietas que irão conferir maior porcentagem de adultos emergidos de $C$. felis felis. Assim, como consequência, haveria maior facilidade na aquisiçáo dos componentes da dieta, além da otimização da manutenção de colônias.

O presente trabalho foi realizado com o objetivo de avaliar a influência de dietas merídicas compostas por sangue desidratado de bovino (Bos taurus), canino (Canis familiaris), galinha (Gallus gallus) e coelho (Oryctolagus cuniculus), acrescidas ou não de farelo de trigo sobre a emergência de adultos da pulga $C$. felisfelis, mantidas em colônia laboratorial.

Este estudo foi realizado no Laboratório de Quimioterapia Experimental em Parasitologia Veterinária, do Departamento de Parasitologia Animal, do Instituto de Veterinária, da Universidade Federal Rural do Rio de Janeiro.

Para a avaliaçáo do percentual de emergência de adultos de C. felis felis, foram preparadas seis dietas merídicas contendo sangue de boi, cão, galinha, e coelho. As amostras de sangue foram desidratadas em estufa a $100{ }^{\circ} \mathrm{C}$, por 24 horas, e, depois de trituradas, foram acrescidas individualmente de farelo trigo em partes iguais, além de areia lavada na proporção de 1:5. Também foram elaboradas dietas com sangue bovino e canino misturados apenas com areia na proporção de 1:20, seguindo-se a metodologia de Correia et al. (2003). Estes autores, utilizando sangue bovino na elaboração de uma dieta merídica, encontraram um percentual de emergência semelhante ao encontrado no ambiente. Diante desse resultado, a dieta elaborada com sangue bovino foi considerada controle no presente estudo.

Foram utilizados 360 ovos de C. felis felis, oriundos da colônia de pulgas mantida nas dependências do laboratório supracitado. Os ovos foram contados e acondicionados em 36 tubos de ensaio, com seis repetiçóes de dez ovos para cada dieta elaborada. Esse procedimento foi realizado com o auxílio de estiletes e microscópio estereoscópico. Os tubos foram acrescidos de $0,5 \mathrm{~g}$ das dietas selecionadas, compostas de sangue de cada espécie animal, farelo de trigo e areia ou somente sangue e areia, na mesma quantidade, e foram devidamente vedados com tecidos de náilon e elástico, sendo posteriormente incubados em câmara climatizada com demanda bioquímica de oxigênio, tipo BOD, $\left(27 \pm 1{ }^{\circ} \mathrm{C}\right.$ e $75 \pm 10 \%$ U.R). Após 25 dias, o conteúdo de cada tubo foi fixado em álcool 70\% e avaliado com o auxílio de microscópio estereoscópico. Os adultos emergidos foram coletados e quantificados. O número médio de adultos obtidos a partir de cada formulação de dieta testada foi comparado pela análise de variância (ANOVA), seguida do teste de Tukey com nível de confiança de 95\% (ZONTA; MACHADO, 1984).

Os resultados obtidos podem ser observados na Tabela 1. A emergência de adultos de $C$. felis felis, cujas larvas foram alimentadas com $0,5 \mathrm{~g}$ de dieta merídica contendo sangue bovino, canino, coelho e galinha, acrescidas de farelo de trigo e areia, foi de oito pulgas. Já para essa mesma quantidade em gramas de dieta, porém, sem o farelo de trigo, essa emergência foi reduzida a uma pulga, para o sangue das espécies canina e bovina.

A emergência de pulgas cujas larvas foram alimentadas com dietas constituídas por sangue de diferentes espécies animais, acrescidas de farelo e areia, não apresentou diferença estatística significante entre si $(\mathrm{p} \leq 0,05)$. Porém, as dietas sem o farelo de trigo, quando preparadas com sangue bovino e canino, diferiram significativamente $(\mathrm{p} \leq 0,05)$ quando comparadas com as demais dietas com farelo de trigo.

De acordo com Bruce (1948), o sangue desidratado a $120^{\circ} \mathrm{C}$ torna-se inadequado para nutrição larval em decorrência de perdas de nutrientes. Alguma perda nutricional também pode ter ocorrido durante o processo de desidratação do presente trabalho, uma vez que a ausência do farelo de trigo que complementaria essa perda, ocasionou um baixo percentual de emergência de adultos de C. felisfelis.

Os resultados do presente trabalho corroboram Correia et al. (2003), que utilizaram, em seus estudos, sangue desidratado de várias espécies animais a $100{ }^{\circ} \mathrm{C}$, comprovando que a adição de farelo de trigo torna-se necessária para o desenvolvimento do ciclo de C. felis felis e proporciona maiores percentuais de emergência, em comparação às dietas sem essa fonte de nutrientes.

No entanto, Moser et al. (1991) obtiveram percentuais de emergência entre 67,5 e $80 \%$, quando empregaram diferentes

Tabela 1. Percentual de emergência de adultos de Ctenocephalides felis felis, oriundos de larvas que se alimentaram de dietas que contêm sangue de diferentes espécies animais, suplementadas ou nấo com farelo de trigo.

\begin{tabular}{cc}
\hline \multicolumn{2}{c}{ Emergência de adultos de Ctenocephalides felis felis } \\
\hline Dietas testadas & Número médio de adultos $\pm \mathbf{d p}$ \\
\hline $\mathrm{BFA}^{1}$ & $7,50 \pm 2,3452 \mathrm{a}$ \\
$\mathrm{BA}^{2}$ & $1,17 \pm 0,9832 \mathrm{~b}$ \\
$\mathrm{CFA}^{3}$ & $8,00 \pm 2,5298 \mathrm{a}$ \\
$\mathrm{CA}^{4}$ & $1,17 \pm 1,6021 \mathrm{~b}$ \\
$\mathrm{CoA}^{5}$ & $7,67 \pm 1,5055 \mathrm{a}$ \\
$\mathrm{GFA}^{6}$ & $8,33 \pm 0,8165 \mathrm{a}$ \\
$\mathrm{F}$ & 26,24 \\
$\mathrm{CV}(\%)$ & 17,75 \\
\hline
\end{tabular}

${ }^{1}$ sangue bovino + farelo de trigo; ${ }^{2}$ sangue bovino; ${ }^{3}$ sangue canino + farelo de trigo; ${ }^{4}$ sangue canino; ${ }^{5}$ sangue de coelho + farelo de trigo; ${ }^{6}$ sangue de galinha + farelo de trigo; Todas as dietas possuem areia em sua composiçáo. Dados originais seguidos do erro padrão da média.

Médias seguidas da mesma letra na coluna não diferem estatisticamente entre si pelo teste de Tukey (5\%)

Teste: One-Way ANOVA. Dados transformados em Raiz quadrada $(x+0,5)$ para fins de análise estatística. F - Teste F; CV - coeficiente de variação 
dietas que contêm sangue bovino, acrescidas ou não de levedura ou ração de roedores. Os autores descrevem que, para desidratação do sangue, utilizaram temperatura de $60{ }^{\circ} \mathrm{C}$, o que pode explicar o fato de que a dieta à base apenas de sangue bovino náo tenha diferido da dieta adicionada de uma fonte de carboidrato. A desidratação a $100{ }^{\circ} \mathrm{C}$ das amostras de sangue utilizadas acarreta alguma perda nutricional, porém, o farelo de trigo pode suprir essas perdas, além de auxiliar na formação do casulo pupal.

Os resultados encontrados demonstram que a espécie animal utilizada para coleta do sangue náo interfere significativamente no percentual de emergência de adultos de $C$. felis felis. Portanto, de acordo com a disponibilidade, é possível a alimentação artificial de larvas, utilizando-se dietas artificiais compostas de sangue de diferentes espécies animais, visando à realização de experimentos in vitro, quando o parâmetro de avaliação utilizado é a emergência de adultos. Entretanto, é importante ressaltar que uma suplementação com farelo de trigo, como fonte de carboidrato, faz-se necessária para suprir a eventual perda nutricional decorrente da desidratação do sangue, além de servir como componente na formação de seu casulo pupal.

\section{Referências}

BRUCE, W. N. Studies on the biological requirements of the cat flea. Annals of the Entomological Society of America, v. 41, n. 3, p. 346-352, 1948.

CORREIA, T. R. et al. Ciclo biológico de Ctenocephalides felis felis (BOUCHÉ, 1835) (SIPHONAPTERA: PULICIDAE) a partir de diferentes dietas artificiais. Revista Brasileira de Zoociências, v. 5, n. 2, p. 153-160, 2003.

HSU, M. H.; HSU, Y. C.; WU, W. J. Consumption of flea faeces and eggs by larvae of the cat flea, Ctenocephalides felis. Medical and Veterinary Entomology, v. 16, n. 4, p. 445-447, 2002.

LAWRENCE, W.; FOIL, L. D. The effects of diet upon pupal development and cocoon formation by the cat flea (Siphonaptera: Pulicidae). Journal of Vector Ecology, v. 27, n. 1, p. 39-43, 2002.

LINARDI, P. M.; MARIA, M.; BOTELHO, J. R. Effects of larval nutrition on the postembryonic development of Ctenocephalides felis felis (Siphonaptera:Pulicidae). Journal of Medical Entomology, v. 34, n. 4, p. 494-497, 1997.

MOSER, B. A.; KOEHLER, P. G.; PATTERSON, R. S. Effect of larval diet on cat flea (Siphonaptera: Pulicidae) developmental times and adult emergence. Journal of Economic Entomology, v. 84, n. 4, p. 1257-1261, 1991.

PÉREZ-OSORIO, C. E. et al. Rickettsia felis as Emergent Global Threat for Humans. Emerging Infectious Diseases, v. 14, n. 7, p. 1019-1023, 2008.

RUST, M. K. Advances in the control of Ctenocephalides felis (cat flea) on cats and dogs. Trends in Parasitology, v. 21, n. 5, p. 232-236, 2005.

VOBIS, M. et al. Evidence of horizontal transmission of feline leukemia virus by the cat flea (Ctenocephalides felis). Parasitology Research, v. 91, n. 6, p. 467-470, 2003.

ZONTA, E. P.; MACHADO, A. A. SANEST: Sistema de Análise Estatísticas para Microcomputadores. Pelotas: UFPel, 1984. 75 p. 